\title{
Influence of teleconnection patterns on precipitation variability and on river flow regimes in the Miño River basin (NW Iberian Peninsula)
}

\author{
Maite deCastro ${ }^{1, *}$, Nieves Lorenzo ${ }^{1}$, Juan J. Taboada ${ }^{2}$, Maria Sarmiento ${ }^{1}$, \\ Ines Alvarez ${ }^{1}$, Moncho Gomez-Gesteira ${ }^{1}$ \\ ${ }^{1}$ Grupo de Física de la Atmósfera y del Océano, Facultad de Ciencias, Universidad de Vigo, 32004 Ourense, Spain \\ ${ }^{2}$ Grupo de Física Non Lineal, MeteoGalicia, University of Santiago de Compostela, 15706 Santiago de Compostela, Spain
}

\begin{abstract}
The influence of the most representative winter patterns of atmospheric variation in the Northern Hemisphere on precipitation variability and on river flow regimes was evaluated for the Miño River basin (NW Iberian Peninsula). The Arctic Oscillation (AO) was also considered in order to analyze the effect of a larger-scale atmospheric mode on precipitation and river flow. Precipitation data were obtained from 22 rain-gauge stations located within the Miño River basin from 1976 to 1997, and the Miño River flow data were recorded at Frieira gauge station from 1970 to 2005. Both hydrological-year (October to September) time series were averaged for the wet season (December to February). The precipitation variability and river flow in the Miño catchment were shown to have high positive correlation. Relationships with atmospheric circulation modes show that the main precipitation variability, and consequently the river-flow regimes, during winter cannot be explained by a single atmospheric pattern. The temporal evolution (1970-2005) of the correlation between the teleconnection indices and river flow averaged for $22 \mathrm{yr}$ periods showed how all atmospheric patterns evolve with a different trend structure-in such a way that the most prevalent pattern depends on the chosen period of time. Moreover, the time evolution of the correlation between the $\mathrm{AO}$ and river flow remains more stationary than for the rest of the indices, due to its larger scale.
\end{abstract}

KEY WORDS: Teleconnection indices $\cdot$ Arctic Oscillation $\cdot$ Precipitation $\cdot$ River flow $\cdot$ Iberian Peninsula

\section{INTRODUCTION}

The maintenance and production of long homogeneous records of river flows is an arduous but essential task for many purposes, such as long-term water resource planning. Flow gauging needs to be continually checked, particularly after major floods or long dry periods, or due to human influences such as water supply or irrigation, which can affect the homogeneity of the series. Series of flow data can be reconstructed using empirical models (Jones \& Lister 1998). Those models transform rainfall to river flow using statistically based catchment models and can be very useful in reconstructing seasonal-mean river flows. On the other hand, annual consumption of energy has contin- uously increased over time in developed countries. This increase has not been followed by a parallel growth in production, which is always limited by technical and environmental constraints. Thus, the understanding and predictability of wind and precipitation variability have been of permanent concern in the scientific community in order to optimize the most important sources of renewable energy (hydroelectric and eolic) to assure sustainable development.

The amount and the distribution of precipitation on the Iberian Peninsula (IP) are highly irregular in both the temporal and spatial dimensions (Serrano et al. 1999, Trigo \& DaCamara 2000). Several authors (Zorita et al. 1992, Rodó et al. 1997, Esteban-Parra et al. 1998, González-Rouco et al. 2000) have investigated the cor- 
respondence between precipitation and the dominant pattern of atmospheric-circulation variability in the North Atlantic, the North Atlantic Oscillation (NAO) (Wallace \& Gutzler 1981). Thompson \& Wallace (1998) introduced the hypothesis that the NAO is the local manifestation of a global pattern called the Arctic Oscillation (AO). This AO is the result of the coupling between the stratospheric polar vortex and tropospheric circulation. Previous research has found a strong correlation between the strength of the polar stratospheric vortex and geopotential anomalies in the middle troposphere in the Northern Hemisphere and that the structure is produced specifically with the NAO pattern. In a later study, Thompson \& Wallace (2000) found that variation in the North Hemispheric polar vortex is stronger in wintertime, and that this is the season when the correlation with the NAO is larger. In this manner, the correlation between the NAO and rainfall or the NAO and river flow in winter could be considered as a consequence of the variations of a hemispheric variation mode (AO). In addition, Rodríguez-Puebla et al. (1998) identified 4 regional precipitation regimes on the IP associated with the following large-scale atmospheric patterns at the monthly time scale: NAO index; East Atlantic (EA); Southern Oscillation Index (SOI) and Scandinavia (SCAND). The NAO consists of a north-south dipole of geopotential anomalies, with one center located over Greenland and the other one spanning between 35 and $40^{\circ} \mathrm{N}$ in the central North Atlantic. A positive phase in the NAO index represents an increase in the pressure differences, resulting in an increase in frequency and strength of winter storms crossing the Atlantic Ocean that typically take a more northerly track. This causes warm and wet winters in northern Europe and drier than normal winters in the south. The negative phase represents a reduced pressure gradient, resulting in fewer and weaker winter storms crossing Europe traveling on a more west-east pathway. This brings moist air into the Mediterranean and causes drier winters in the northern part of Europe. The EA pattern consists of a north-south dipole that spans the entire North Atlantic Ocean, with the centers near $55^{\circ} \mathrm{N}, 20-35^{\circ} \mathrm{W}$ and $25-35^{\circ} \mathrm{N}, 0-10^{\circ} \mathrm{W}$. The anomaly centers of the EA pattern are displaced southeastward of the approximate centers of the NAO pattern. The positive phase of the EA is associated with above-average precipitation over northern Europe and Scandinavia and with below-average precipitation across southern Europe (Rodríguez-Puebla et al. 1998, 2001). On the other hand, the positive phase of the EA pattern is associated with above-average surface temperatures in Europe. In particular in the northern part of the IP, the EA pattern is the most important variation pattern explaining temperature variability (Sáenz et al.
2001). The SOI is calculated from the monthly or seasonal fluctuations in the air-pressure difference between Tahiti and Darwin. Sustained negative values of the SOI often indicate El Niño episodes. Positive values of the SOI are associated with stronger Pacific trade winds and indicate a La Niña episode. The SOI has been shown to influence rainfall variability over the eastern part of the IP in winter (Rodó et al. 1997, Rodríguez-Puebla et al. 2001). The SCAND pattern consists of a primary circulation center over Scandinavia, with a weaker center of opposite sign over western Europe. The positive phase of this pattern is associated with positive height anomalies, sometimes reflecting major blocking anticyclones, over Scandinavia and western Russia. The East Atlantic/West Russia (EA/WR) pattern (Barnston \& Livezey 1987) is one of 3 prominent teleconnection patterns that affect Eurasia through the year. This pattern consists of 4 main anomaly centers. The positive phase is associated with positive height anomalies located over Europe and northern China, and negative height anomalies located over the central North Atlantic and north of the Caspian Sea. The main precipitation departures associated with the positive phase of the EA/WR pattern reflect generally aboveaverage precipitation in eastern China and belowaverage precipitation across central Europe. The Europe Polar/Eurasia (POL) pattern, the most prominent index from December through February (DJF), consists of one center over the polar region and centers of opposite sign over Europe and northeastern China.

Many authors have pointed out that several atmospheric patterns influence precipitation in the northwest part of the IP, but that no one pattern describes the majority of the variability in precipitation over the region. Zorita et al. (1992) and Esteban-Parra et al. (1998) showed correlation coefficients between the NAO index (DJF) and rainfall of -0.3 to -0.4 in Galicia. Other work by Rodríguez-Puebla et al. (1998) found a correlation coefficient between the December NAO index and the annual precipitation of -0.10 to -0.15 . In the same analysis the authors found correlation coefficients with the annual rainfall: 0.3 to 0.4 for the April EA pattern; 0.05 to 0.1 for the October of the previous year's SOI; and 0.35 to 0.4 for the December SCAND. In addition, these correlation coefficients have been shown to be non-stationary, changing in magnitude during the twentieth century (Rodó et al. 1997, Goodess \& Jones 2002).

Several studies show the strong influence of the NAO on precipitation over the Mediterranean basin that is directly reflected in the seasonal flow of rivers across the region such as the Tigris, Euphrates and Jordan (e.g. Cullen et al. 2002). Each of these studies has shown how river flow tends to be lower (higher) when the NAO is in its positive (negative) phase. The 
precipitation variability on the IP, as previously mentioned, produces large inter-annual variations in the river-flow regimes of all IP rivers. Trigo et al. (2004) found that the influence of the NAO on IP river-flow regimes is larger than it is on the Danube or Middle Eastern rivers. They analyzed the temporal evolution of the correlation coefficient between the NAO index for the wet season on river basin precipitation and river flow for 3 Iberian river basins (Douro, Tagus and Guadiana) throughout most of the twentieth century. According to the OSPAR commission (www.ospar.org), IP rivers are characterized by their inter-annual variability in run-off, which is close to $85 \%$ for the Guadiana basin, decreasing northward to $55 \%$ for the Tagus, $53 \%$ for the Douro and $40 \%$ for the Miño. Galician rivers in general, and the Miño River in particular, are especially important in terms of discharge compared to the basin extent. The ratio between run-off and catchment area (in $\mathrm{Hm}^{3} \mathrm{yr}^{-1} \mathrm{~km}^{-2}$ ) is 0.75 for the Miño River, 0.23 for the Douro, 0.17 for the Tagus and 0.08 for the Guadiana (www.ospar.org).While Galicia represents only about $6 \%$ of the area of Spain, it provides about $20 \%$ of the hydroelectric energy, although with a considerable inter-annual variability $(6332 \pm 720 \mathrm{GWh}$ during 1999-2004 according to Red Electrica de España, www.ree.es). This level of precipitation variability, which can produce dramatic effects in terms of energy production, can likely be partially mitigated by predicting winter teleconnection patterns. The ability to forecast Galician winter rainfall could eventually be used for planning purposes. As an example, it has been found that the NAO can be predicted with reasonable skill from North Atlantic sea-surface-temperature anomalies averaged over several prior months (Saunders \& Budong 2002, Czaja \& Marshall 2002, Bojariu \& Gimeno 2003a,b). On the other hand, interannual variations in snow mass in the Northern Hemisphere have been found to exert a modulating influence on the NAO index (Cohen \& Entekhabi 1999, Bojariu \& Gimeno 2003). These 2 mechanisms could be used to make a seasonal winter forecast of the NAO, which in turn could improve the seasonal winter rainfall forecast, and in turn predict the river-flow regimes for Galicia.

The aim of this paper is to analyze the relationships between the most representative winter patterns of atmospheric variability in the Northern Hemisphere (NAO, SCAND, EA, EA/WR and POL) and the precipitation variability and the river flow regime for the Miño River basin from 1976 to 1997 . The AO was also considered to account for the effect of a larger-scale atmospheric mode. This study was carried out for the wet season (DJF). In addition, the temporal evolution of the correlation between the atmospheric patterns and river flow was analyzed in 22 yr periods from 1970 to 2005 .

\section{THE MIÑO RIVER BASIN}

The Miño River, located at the northwest corner of the IP, rises in Serra Meira (Galicia) and acts as a natural border between Spain and Portugal over its last $75 \mathrm{~km}$ (Fig. 1), flowing into the Atlantic Ocean in A Guarda. The length of the river is approximately $307.5 \mathrm{~km}$.

The annual precipitation in the mountains located in the northwestern provinces of Minho (Portugal) and Galicia (Spain) often exceed values of $2500 \mathrm{~mm} \mathrm{yr}^{-1}$. Most of the precipitation falls between October and May, and it is produced by synoptic-scale baroclinic perturbations that originate in the Atlantic sector and move eastward (Serrano et al. 1999). During winter, the large-scale circulation is mainly driven by the position and the intensity of the Iceland Low, and western Iberia is affected by westerly winds that carry moist air and produce rainfall events. This precipitation is intensified by the passage of cold fronts associated with transient depressions. This mechanism is particularly efficient when the Iceland Low is very deep and shifted southwards (Trigo et al. 2004).

The annual variability of the hydrologic cycle for the Miño River is represented in the monthly average of the river discharge (Fig. 2). The mean monthly flow oscillates between maximum runoff, during the wet season (DJF), and minimum runoff, which occurs during the dry season (JAS).

\section{DATA AND METHODS}

Monthly precipitation data from 1976-1997 were obtained from the database CLIMA of the University of Santiago de Compostela for the 22 rain-gauge stations of the National Institute of Meteorology (INM) that are distributed throughout the Miño River basin (Fig. 1). The CLIMA database has been submitted to quality controls and has been checked for inhomogeneities. The characteristics of the rain-gauge stations are summarized in Table 1. For this analysis, the precipitation data were averaged for the winter period (DJF) corresponding to the wet season during hydrological years (October-September). Only stations with more than $80 \%$ of the monthly precipitation data processed were considered, in order to avoid bias in results. Although orography and distance are not likely to alter conditions from one station to another due to the limited extent of the Miño River basin, data were spatially integrated over the basin by means of a weighted average procedure following Alexander \& Jones (2001).

The teleconnection indices NAO, SCAND, EA, EA/WR and POL and the atmospheric mode AO, at monthly time scales, were obtained from the Climate 


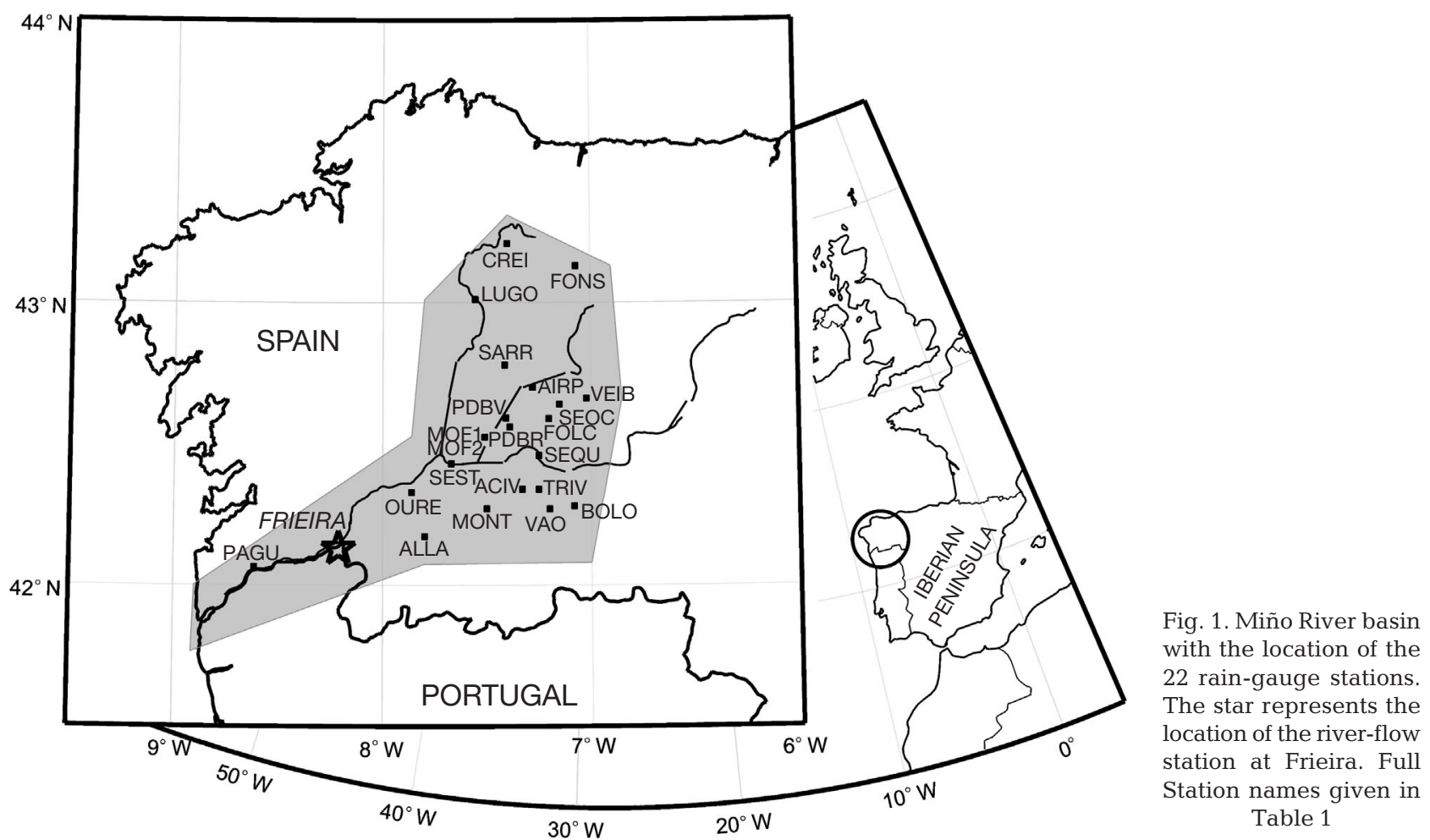

Prediction Center (CPC) at the National Center of Environmental Prediction (NCEP; www.cpc.noaa.gov). The monthly indices were averaged for the same DJF period from 1970 to 2005. The most representative atmospheric patterns of the Northern Hemisphere were considered, in order to analyze their influence on precipitation and river flow for the region. Research has identified the NAO index as the local manifestation of the more global pattern of the AO during the winter (DJF) (Thompson \& Wallace 1998, 2000, Wallace 2000, Ambaum et al. 2001). Although the correlations between the teleconnection indices and the AO on a global scale is far beyond the aim of the present study, the correlations between the atmospheric patterns (NAO, SCAND, EA, EA/WR and POL) and the AO were considered during DJF for the period under study (1976-1997). During this period, the NAO and AO indices were very well correlated with a correlation coefficient of 0.76 . In addition, the $\mathrm{AO}$ and SCAND revealed strong a negative correlation, with a value of -0.81 . The correlations between the $\mathrm{AO}$ and the rest of the teleconnection indices were negligible.

Monthly river discharge data from October 1970 to September 2005 were obtained from the Confederación Hidrográfica del Norte at a gauge station situated in Frieira with a river basin area of approximately $15864 \mathrm{~km}^{2}$ (Fig. 1). The river discharge was averaged for the same DJF period corresponding to the wet season during hydrological years (October-September).

\section{RESULTS AND DISCUSSION}

The correlation between precipitation and river flow and the time lags between both variables were studied from 1976 to 1997 . The inter-annual variability of the precipitation and the Miño River flow is represented in Fig. 3. In order to facilitate a visual comparison, both variables were normalized $\left(x_{i}^{n}=\left(x_{i}-\bar{x}\right) / \sigma(x)\right.$, where $x_{i}^{n}$ refers to normalized values and $\sigma(x)$ is the standard

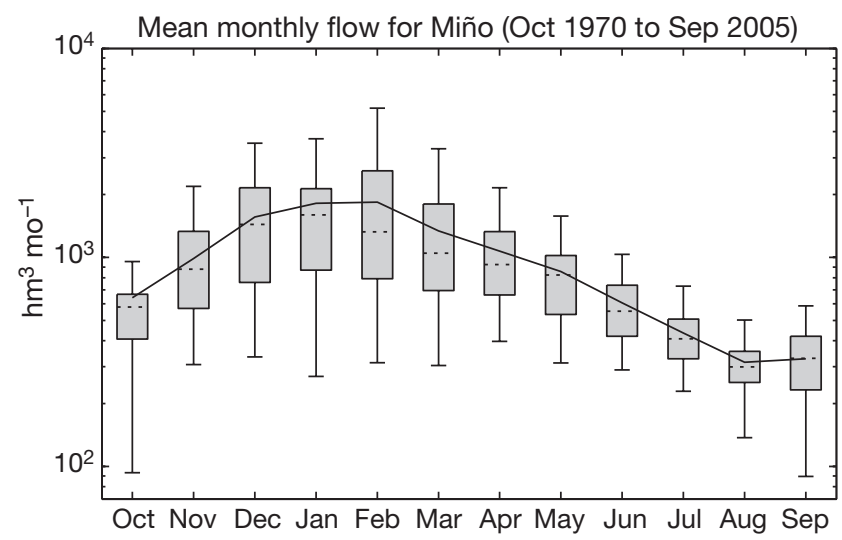

Fig. 2. Annual hydrologic cycle variability for the monthly average Miño River flow from 1970 to 2005. Solid line represents the monthly average, dotted line inside each box represents the mean value for each month, the lower/upper whisker represents the minimum/maximum river flow and the lower/ upper box limits represent the first/third quartile, respectively 
Table 1. Location and DJF average for precipitation from the 22 rain-gauge stations located within the Miño River basin

\begin{tabular}{|c|c|c|c|c|}
\hline Station & Longitude (W) & Latitude (N) & Elevation (m) & DJF average precipitation $(\mathrm{mm})$ \\
\hline Fonsagrada (FONS) & $7^{\circ} 03^{\prime}$ & $43^{\circ} 07^{\prime}$ & 952 & 372.8 \\
\hline Montederramo (MONT) & $7^{\circ} 30^{\prime}$ & $42^{\circ} 16^{\prime}$ & 900 & 619.2 \\
\hline Folgoso De Caurel (FOLC) & $7^{\circ} 11^{\prime}$ & $42^{\circ} 35^{\prime}$ & 612 & 607.4 \\
\hline VAO (VAO) & $7^{\circ} 09^{\prime}$ & $42^{\circ} 15^{\prime}$ & 711 & 421.9 \\
\hline O Bolo (BOLO) & $7^{\circ} 05^{\prime}$ & $42^{\circ} 18^{\prime}$ & 798 & 301.0 \\
\hline Monforte De Lemos 'E. Agricola' (MOF1) & $7^{\circ} 30^{\prime}$ & $42^{\circ} 31^{\prime}$ & 363 & 308.3 \\
\hline Castro De Rei (CREI) & $7^{\circ} 23^{\prime}$ & $43^{\circ} 12^{\prime}$ & 439 & 437.5 \\
\hline San Esteban (SEST) & $7^{\circ} 40^{\prime}$ & $42^{\circ} 26^{\prime}$ & 180 & 456.5 \\
\hline Aira Padron (AIRP) & $7^{\circ} 16^{\prime}$ & $42^{\circ} 41^{\prime}$ & 1100 & 688.7 \\
\hline Puebla De Brollon (PDBR) & $7^{\circ} 23^{\prime}$ & $42^{\circ} 33^{\prime}$ & 401 & 349.4 \\
\hline Seoane Do Courel (SEOC) & $7^{\circ} 09^{\prime}$ & $42^{\circ} 38^{\prime}$ & 661 & 583.8 \\
\hline Veiga De Brañas (VEIB) & $7^{\circ} 01^{\prime}$ & $42^{\circ} 39^{\prime}$ & 1150 & 670.4 \\
\hline Sequeiros (SEQU) & $7^{\circ} 15^{\prime}$ & $42^{\circ} 27^{\prime}$ & 280 & 342.5 \\
\hline Aciveiros (ACIV) & $7^{\circ} 19^{\prime}$ & $42^{\circ} 20^{\prime}$ & 978 & 443.9 \\
\hline Trives 'San Juan Del Rio' (TRIV) & $7^{\circ} 15^{\prime}$ & $42^{\circ} 20^{\prime}$ & 761 & 388.7 \\
\hline Paramos-Guillarei (PAGU) & $8^{\circ} 36^{\prime}$ & $42^{\circ} 3^{\prime}$ & 45 & 680.6 \\
\hline Orense 'Granja Diputacion' (OURE) & $7^{\circ} 51^{\prime}$ & $42^{\circ} 19^{\prime}$ & 150 & 302.0 \\
\hline Monforte 'A Pinguela' (MOF2) & $7^{\circ} 32^{\prime}$ & $42^{\circ} 31^{\prime}$ & 283 & 252.2 \\
\hline Puebla De Brollon 'Veiga' (PDBV) & $7^{\circ} 24^{\prime}$ & $42^{\circ} 35^{\prime}$ & 400 & 389.5 \\
\hline Sarria 'Granxa Barreiros' (SARR) & $7^{\circ} 24^{\prime}$ & $42^{\circ} 46^{\prime}$ & 550 & 475.0 \\
\hline Allariz (ALLA) & $7^{\circ} 48^{\prime}$ & $42^{\circ} 10^{\prime}$ & 766 & 329.8 \\
\hline Lugo 'Colegio Fingoi' (LUGO) & $7^{\circ} 33^{\prime}$ & $43^{\circ} 00^{\prime}$ & 450 & 371.1 \\
\hline
\end{tabular}

deviation of variable $x_{i}$ ). The 2 variables show great correspondence from 1976 to 1983 and from 1989 to 1997. From 1984 to 1988 both patterns are not as strongly related but follow the same trend. The correlation coefficient between river flow and precipitation is 0.88 , with a significance level greater than $99 \%$. In order to analyze the time lag between the DJF precipitation and the Miño River flow, the data was temporally averaged for different time periods and is shown

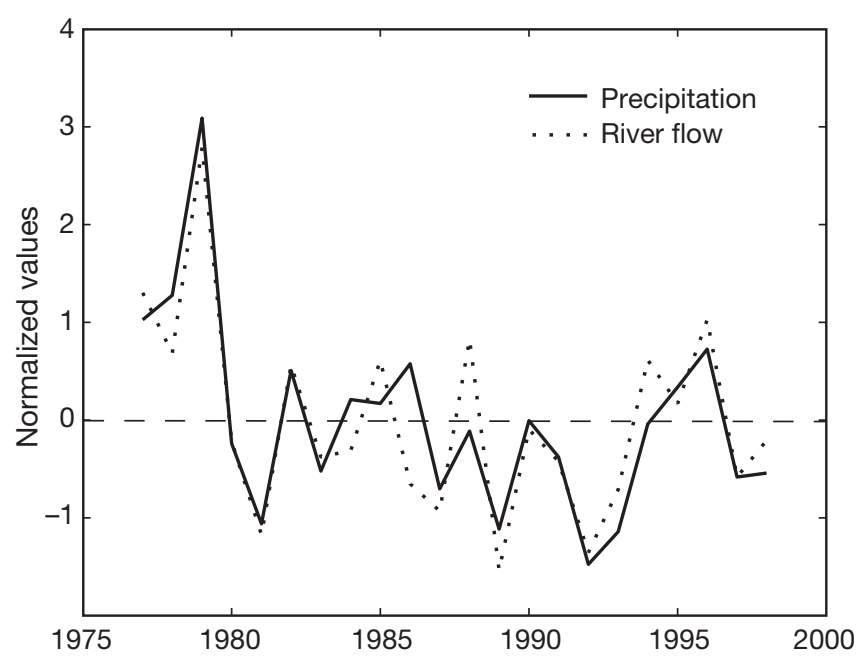

Fig. 3. Inter-annual variability of DJF precipitation (spatially integrated over the Miño River basin; solid line) and DJF river flow (dotted line) from October 1976 to September 1997. The precipitation and river flow data were normalized in order to facilitate visual comparison in Fig. 4. The correlation coefficient is close to 0.9 for time lags of 0, 1 and even 2 mo. This coefficient drops to values under 0.6 for time lags longer than 3 mo.

The correlation coefficients between DJF atmospheric patterns and river flow (temporally averaged for different time periods) are also given in Fig. 4. The EA index is shown to be directly correlated with river flow with a correlation coefficient close to 0.4 . This correlation coefficient drops continuously when any greater time lag is considered. The correlation coefficients between river discharge and the SCAND and EA/WR indices show a different behaviour. In these 2 cases the highest correlation coefficient $(0.61$ and -0.54 , respectively) are achieved for a lag of 1 mo (river flow averaged for the period JFM). For time lags longer than 3 mo, both correlation coefficients drop continuously. In contrast, the correlation coefficient between river flow and the NAO index achieves its maximum value of -0.66 for a time lag of 2 mo. This result contrasts with those obtained by Trigo et al. (2004) for other Atlantic Iberian rivers. In their results, the correlation between the DJF NAO index and river flow increased when a time lag of $1 \mathrm{mo}$ is considered. In addition, the $\mathrm{AO}$ is shown to be better correlated with river flow when 1 or 2 mo of time lag is considered $(-0.64)$, following a behaviour similar to that of the NAO index.

The inter-annual variation of the atmospheric indices (AO, NAO, EA, EA/WR and SCAND) and precipitation spatially integrated over the Miño catchment from 1976 to 1997 is given in Fig. 5. The inter-annual variation of the AO index is shown in the same frame as the 


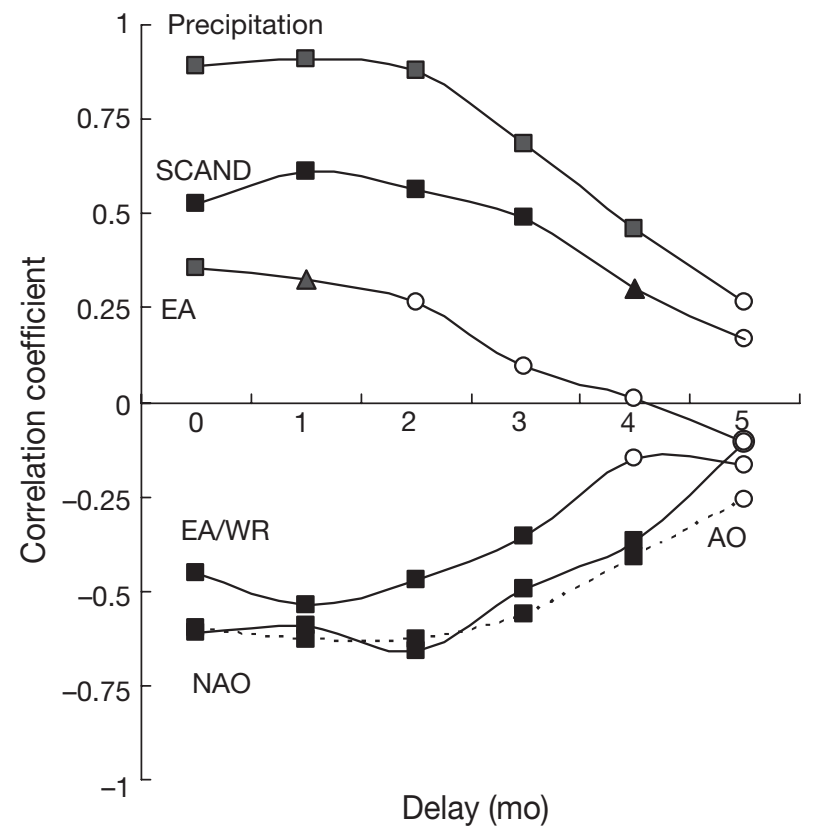

Fig. 4. DJF precipitation (spatially integrated over the Miño River basin) and DJF atmospheric patterns correlations with river flow (temporally averaged for different time periods) from 1976 to 1997. A 0 corresponds to the DJF river-flow period, 1 to JFM period and so on. Results with a significance level greater than $95 \%$ are represented by squares, greater than $90 \%$ by triangles, and less than $90 \%$ by empty circles

NAO index due to the similarity between the two atmospheric patterns. To facilitate visual comparison the same protocol as in Fig. 3 was used to normalize the precipitation values. During the time period, the indices appear to fit the precipitation variability most accurately during specific periods (NAO and EA from 1976 to 1983, AO from 1983 to 1997, EA/WR from 1991 to 1997 and SCAND from 1979 to 1997).

The correlations between the teleconnection patterns and precipitation were also analyzed at each rain-gauge station for the Miño River basin (Fig. 1) from 1976 to 1997 (Fig. 6). The highest correlations were obtained for the NAO index, ranging from -0.48 to -0.71 . The correlation coefficients between rainfall and the EA/WR index ranged from -0.2 to -0.6 and between rainfall and the EA and SCAND indices ranged from 0.25 and 0.6 , showing a similar influence of the 3 teleconnection indices on the precipitation pattern. Finally, no correlation between POL and rainfall was observed.

Table 2 summarizes the influence of the atmospheric patterns on precipitation by showing the mean and standard deviation of the correlations calculated at each rain-gauge station. These correlations are significant only for the AO, NAO, SCAND, EA/WR and EA indices with a significance level that exceeds $95 \%$. These results reveal some of the features mentioned above, namely, the NAO index has the greatest influence on precipitation for this region. Note that the NAO and AO indices show a similar correlation, which agrees with the other observations that the NAO is a local manifestation of the AO (Thompson \& Wallace 1998, 2000). The rest of the indices-SCAND, EA/WR and EA - show a lower, although significant, correlation with precipitation. Galicia is characterized by the passage of cold fronts associated with lows traveling across the North Atlantic. The storm track in this area depends strongly on the NAO state during the winter,
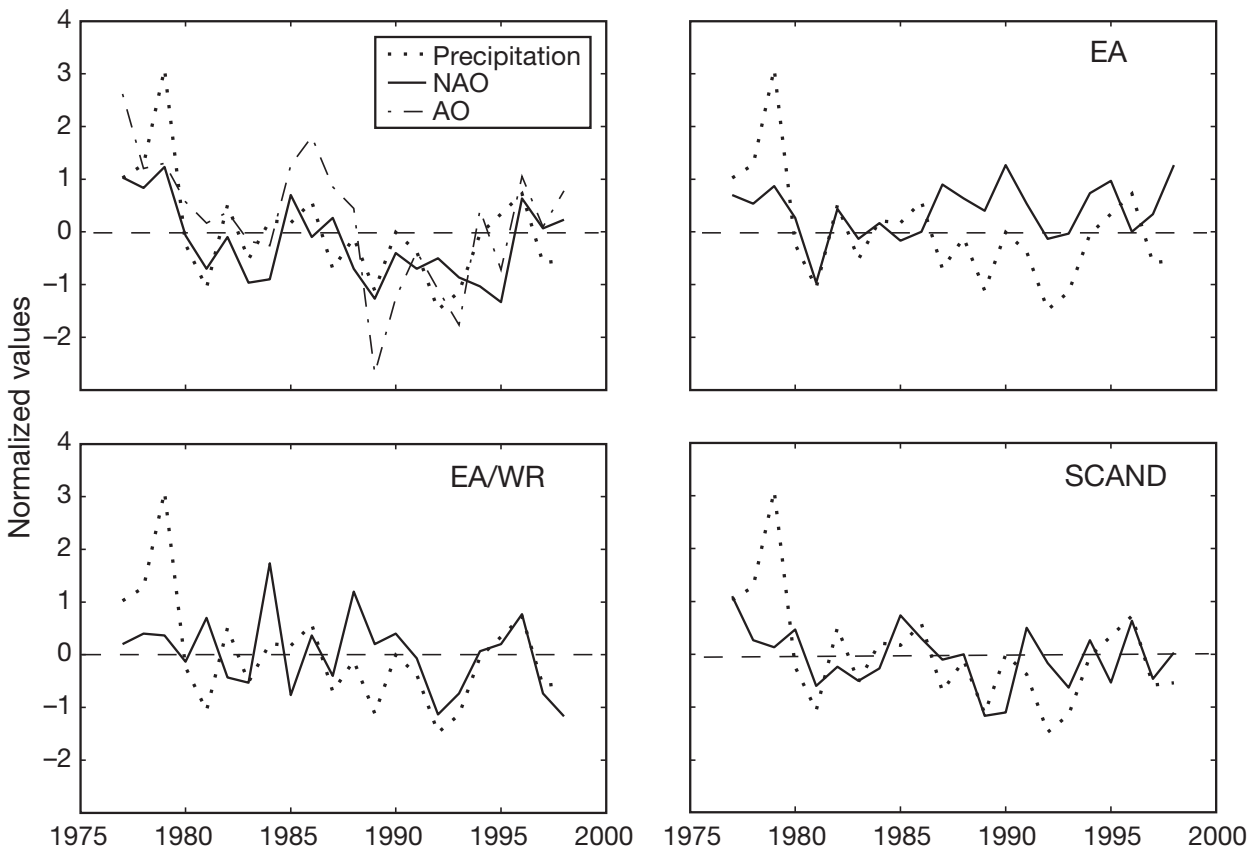

Fig. 5. Inter-annual variation of DJF atmospheric patterns (AO, NAO, EA, EA/WR and SCAND; solid line) and DJF precipitation (dotted line) (spatially integrated over the Miño River basin) from October 1976 to September 1997. The AO, NAO and the EA/WR indices were multiplied by -1 , and the precipitation data were normalized to facilitate visual comparison 

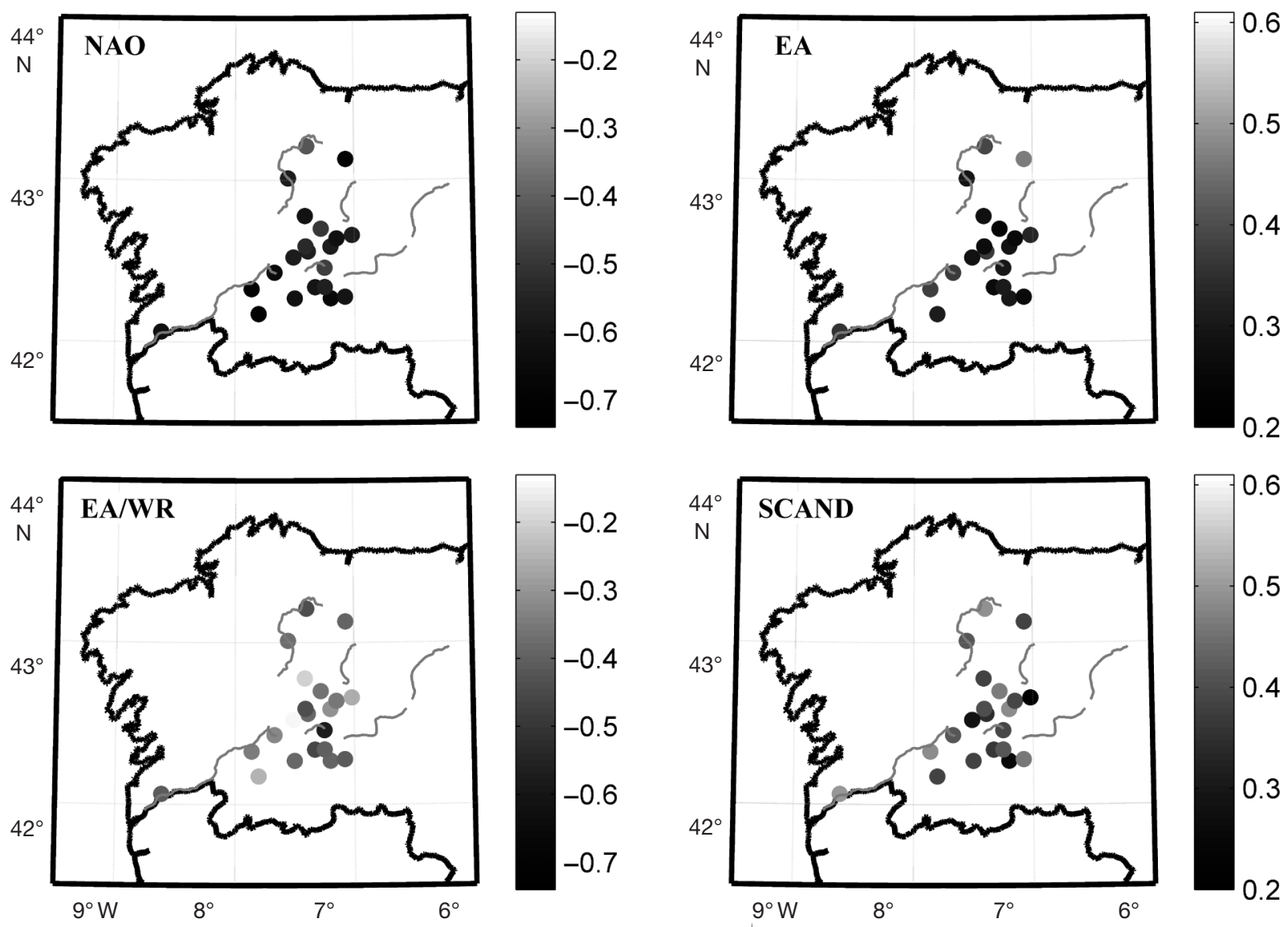

Fig. 6. Correlation coefficients between DJF teleconnection indices (NAO, EA, EA/WR and SCAND) and DJF precipitation at each rain-gauge station from October 1976 to September 1997

which will make the passage of cold fronts easier or more difficult. On the other hand, the action centers of the north-south dipole of the EA are located to the southwest of the NAO dipole. Considering that Galicia is strongly influenced by the NAO, but in an area near the latitude where influences pass from positive to negative correlations, it is clear that the EA must have an important influence on winter rainfall as well. In this manner a positive EA pattern means that subtropical high and lows in the North Atlantic are stronger.

Table 2. Mean and standard deviation of the correlation coefficients between DJF rainfall and DJF atmospheric patterns calculated at each rain-gauge station

\begin{tabular}{|cr|}
\hline Index & Correlation \\
\hline AO & $-0.56 \pm 0.02$ \\
NAO & $-0.59 \pm 0.02$ \\
SCAND & $0.39 \pm 0.02$ \\
EA/WR & $-0.36 \pm 0.02$ \\
EA & $0.33 \pm 0.02$ \\
POL & $-0.053 \pm 0.02$ \\
\hline
\end{tabular}

The northern part of the dipole is located in a latitude band between Newfoundland and Ireland in the North Atlantic. Therefore, a positive EA pattern means that low pressure will dominate over this area, and in this situation, more cold fronts will reach the Galician coast during the winter. In addition, the positive state of the east-west dipole of SCAND produces a negative pressure center over the IP, forcing the storm track to approach the latitude of Galicia (located at a crossing point, with Atlantic but also continental influences), even if neither NAO nor EA favor this trajectory. The EA/WR features in their positive phases imply the existence of high-pressure centers located over Europe, mainly to the north of the IP. This location prevents the jet stream from traversing southward toward Galicia, typically passing to the north or to the south, causing more rain in Scandinavia and the Canary Islands and leaving conditions dry over the IP, which explains the negative correlation with winter rainfall in Galicia. On the other hand, the POL atmospheric pattern has a negligible influence on the precipitation pattern over the Miño River basin. This index represents a 
north-south dipole, as with the NAO and EA, but with the centers of action located farther north than the NAO centers. Thus, variations in this index do not favor a correlation in regions located below $40^{\circ} \mathrm{N}$.

As mentioned previously, the influence of the atmospheric indices on the precipitation variability in the IP can be extended to the flow of the Iberian rivers. For example, Trigo et al. (2004) found correlation coefficients between the DJF-averaged NAO index and the JFM-averaged river flow for 1923-1998 of -0.55 for the Douro River, -0.52 for the Tagus River and -0.69 for the Guadiana River. The correlation coefficients are stronger from south to north according to other research (Zorita et al. 1992, Rodó et al. 1997, RodríguezPuebla et al. 1998, 2001), where the highest correlation coefficients between the December NAO index and the annual precipitation were observed in the southwestern corner of the IP. The correlations are considerably larger when 1973-1998 is considered: -0.76 for the Douro River, -0.77 for the Tagus River and -0.79 for the Guadiana River, which shows the lack of stationarity in the correlation (Goodess \& Jones 2002).

The inter-annual variation of the DJF-averaged teleconnection indices and the winter Miño River flow are given for 1976 to 1997 in Fig. 7. A time lag of 0 (DJF), 1 (JFM) or 2 (FMA) mo is considered in the Miño River flow, depending on the teleconnection index according to Fig. 4. The inter-annual variation of the $\mathrm{AO}$ index is once again given in the same frame as the NAO index due to the similarity between the two atmospheric indices. In order to facilitate visual comparison, the same protocol as in Fig. 5 was used, and the following correlation coefficients were found: -0.66 for the NAO index, -0.64 for the AO index, 0.61 for the SCAND, -0.54 for the EA/WR, 0.36 for the EA and -0.11 for the POL index. These correlations are significant for the AO, NAO, SCAND, EA/WR and EA indices with a significance level that exceeds $95 \%$. The influence of the SCAND atmospheric pattern has the same magnitude as that of the NAO, with the influence of the EA and EA/WR atmospheric indices also significant.

In order to compare the correlation between the NAO index and the river flow for the Miño River with the other Atlantic IP rivers, the period of time considered by Trigo et al. (2004) (from 1973 to 1998) was used. The results show correlation coefficients of -0.65 for the NAO and AO indices, 0.64 for the SCAND, -0.50 for the EA/WR, 0.44 for the EA and -0.13 for the POL index. The correlation coefficient obtained between the Miño River flow and the NAO index is smaller than the one obtained for the Douro River, which follows the tendency for correlations to diminish from south to north over the region. In this case the highest correlation between the NAO index and river flow was achieved for a time lag of $2 \mathrm{mo}$, which contrasts with the results obtained by Trigo et al. (2004), in which the highest correlation was obtained for a time lag of 1 mo. This is possibly due to the orographic constraints and the high density of dams located in the Miño River basin compared with the rest of Iberian rivers.

The non-stationarity of the atmospheric indices becomes more apparent when comparing the correlation with river flow for both time periods. An increase in the SCAND and EA correlation coefficients and a decrease in the EA/WR correlations are observed from 1976-1997 to 1973-1998. In contrast, the NAO correla-
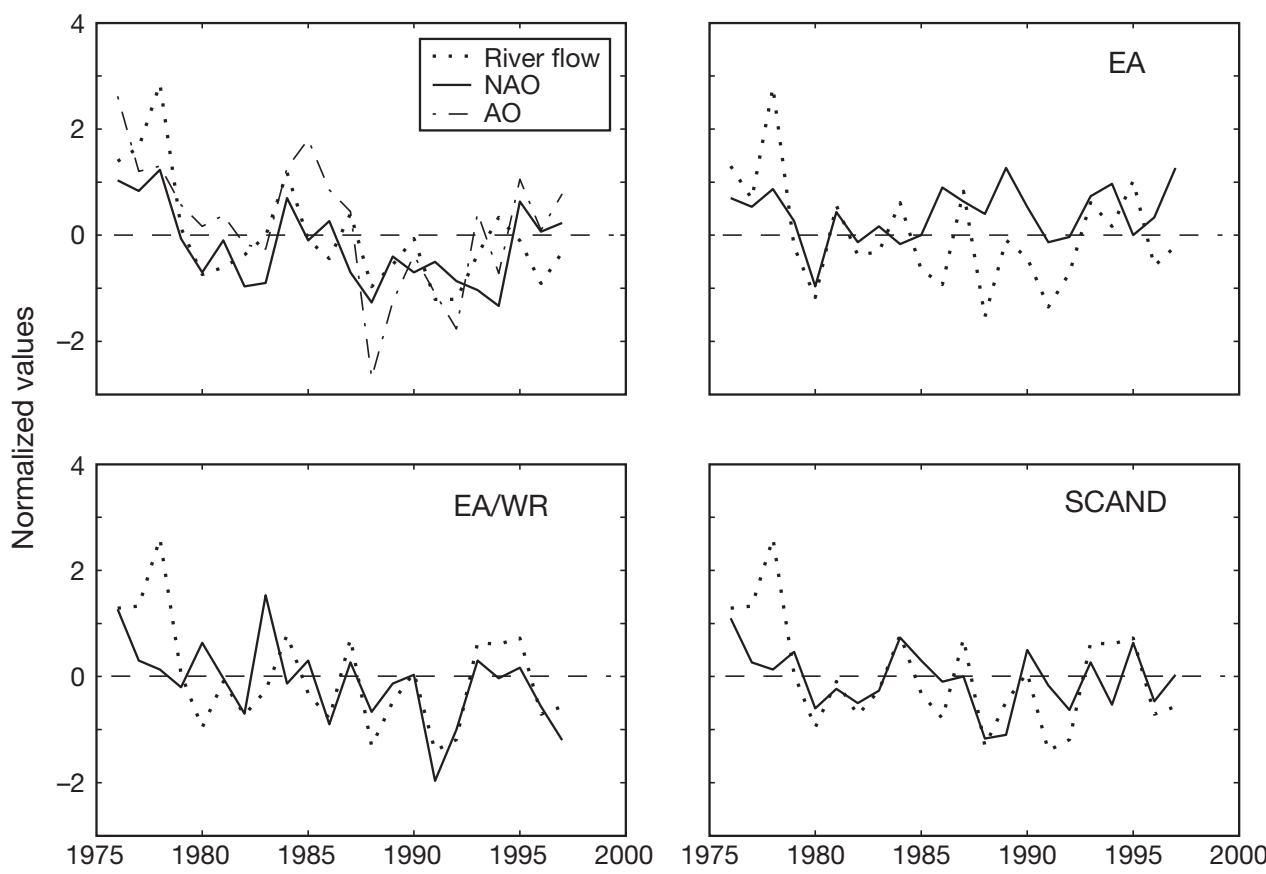

Fig. 7. Inter-annual variability of DJF atmospheric patterns (AO, NAO, EA, EA/WR and SCAND; solid line) and DJF river flow (dotted line) from October 1976 to September 1997. A river flow time lag of 0 (EA), 1 (SCAND and EA/WR) and 2 (NAO and AO) mo was considered, depending on the atmospheric pattern. The AO, NAO and the EA/WR indices were multiplied by -1 , and the river flow data were normalized to facilitate visual comparison 
tion coefficient remains nearly constant. Therefore, the atmospheric patterns evolve temporally with different structures, in such a way that the most prevalent pattern depends on the chosen period of time. The time evolution of the correlation coefficients between the atmospheric indices $(\mathrm{AO}, \mathrm{NAO}, \mathrm{EA}, \mathrm{EA} / \mathrm{WR}$ and SCAND) and river flow averaged for $22 \mathrm{yr}$ periods (duration of the precipitation data) from 1970 to 2005 are represented in Fig. 8. The mean and standard deviation of these correlation coefficients are summarized in Table 3. Both Fig. 8 and Table 3 show the significance of the NAO, SCAND, EA/WR and EA atmospheric patterns in explaining the inter-annual variability of the Miño River flow. In particular, it is clear that the NAO pattern has the greatest influence on Miño River flow from 1970-1991 to 1976-1997, with correlation coefficients close to -0.7 . Afterwards, the correlation coefficient decreases, reaching values below -0.4 for the last 5 periods. In contrast, the correlation coefficient between the SCAND pattern and Miño River flow increases during the entire time period, reaching the highest correlation values (close to 0.7 ) for the last 5 periods. In addition, the results show how the decrease in the NAO correlation coefficient also coincides temporally with the decrease in the EA/WR and EA correlation coefficients. The winter NAO exhibits significant multi-decadal variability, where the negative phase of the NAO (which is the phase with the most influence on the Miño River basin) dominated the circulation from the mid-1950's through to the 1978/79 winter (Hurrell 1995). During this approximately $24 \mathrm{yr}$ interval, there were 4 prominent periods, of at least $3 \mathrm{yr}$ each, in which the negative phase was dominant and the positive phase was notably absent. In fact, during the entire period the positive phase was observed in the seasonal mean only 3 times, and never in 2 consecutive years. This explains the high correlation coefficient between NAO pattern and Miño River flow and the relatively small influence of the SCAND pattern. An abrupt transition to recurring positive phases of the NAO occurred during the
1979/80 winter, with the atmosphere remaining locked near these index values through the 1994/95 winter season. During this 15 yr interval, a substantial negative phase of the pattern appeared only twice, in the winters of 1984/85 and 1985/86. Thus the NAO positive phase shows less influence on precipitation in Galicia and consequently on the Miño River flow, with SCAND becoming the more relevant circulation pattern influence.

The time evolution of the correlation between the $\mathrm{AO}$ and the river flow shows a continuous decrease for the whole time period. This decrease is more gradual than either the NAO decrease or the increase in the correlation coefficient between the SCAND index and river flow. Thus, the correlation with AO remains more stationary than the correlations for the rest of the indices. This result shows the dependence on the larger scale of the AO.

\section{CONCLUSIONS}

Galicia is strongly influenced by the NAO, but is located on the Atlantic side of Europe near the latitude where the influences pass from positive to negative correlations. For this reason, the main variability of precipitation and river-flow regimes in Galicia are best explained by means of 4 atmospheric patterns (NAO,

Table 3. Mean \pm standard deviation of the simultaneous (lead 0 ) and leading correlation coefficients between the DJF atmospheric patterns and Miño River flow averaged for $22 \mathrm{yr}$ periods from 1970 to 2005

\begin{tabular}{|lrrc|}
\hline Index & \multicolumn{2}{c|}{$\begin{array}{c}\text { Correlation } \\
\text { (lead 0) }\end{array}$} & \multicolumn{1}{c|}{$\begin{array}{c}\text { Lead } \\
\text { (with lead) }\end{array}$} \\
\hline AO & $-0.59 \pm 0.01$ & $-0.63 \pm 0.02$ & 1 and 2 (JFM, FMA) \\
NAO & $-0.51 \pm 0.04$ & $-0.54 \pm 0.05$ & 2 (FMA) \\
SCAND & $0.54 \pm 0.02$ & $0.63 \pm 0.01$ & 1 (JFM) \\
EA/WR & $-0.39 \pm 0.02$ & $-0.46 \pm 0.02$ & 1 (JFM) \\
EA & $0.40 \pm 0.02$ & $0.40 \pm 0.02$ & 0 (DJF) \\
\hline
\end{tabular}

Fig. 8. Time evolution of the correlation coefficients between DJF atmospheric patterns (AO, NAO, EA, EA/WR and SCAND) and the Miño River flow, averaged for $22 \mathrm{yr}$ periods from 1970 to 2005. River-flow time lags of 0 (EA), 1 (SCAND and EA/WR) and 2 (NAO and AO) mo were considered, depending on the atmospheric pattern

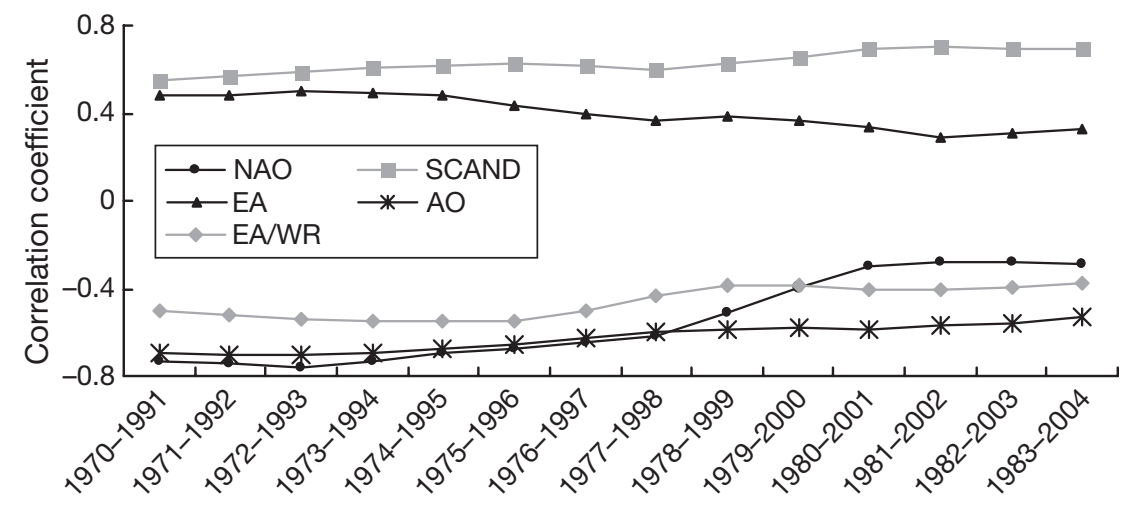


SCAND, EA and EA/WR). The NAO is observed to be the most prominent teleconnection index, followed by the SCAND pattern. The EA/WR and EA atmospheric patterns are also significant. In contrast, the fifth atmospheric pattern analyzed (POL) does not show any significant correlation, whereas the AO mode presents a high correlation, similar to the ones obtained with NAO and SCAND. It was also observed that winter rainfall and river flow have a correlation coefficient of 0.88 for the Miño River basin. This correlation coefficient remains almost constant when considering a time lag of 1 or 2 mo between precipitation and river flow.

Inter-annual variation of the correlation coefficients between teleconnection indices and river flow from 1970 to 2005 reveals how each atmospheric pattern follows a different trend structure, in such a way that the most prevalent index depends on the chosen period of time. The results show that from 1970-1991 to 1976-1998 the NAO pattern has the most influence on Miño River flow, coinciding with a negative phase of the NAO domination of the circulation. The SCAND pattern shows its dominance in later time periods, which coincide with positive phases of the NAO index. The time evolution of the correlation between the AO and river flow remains more stationary due to its larger scale. By comparing this with previous studies, it was observed that the correlation coefficient obtained between the FMA Miño River flow and the DJF NAO index from 1973 to 1998 follows the decreasing trend from south to north observed in other Iberian rivers (e.g. the Guadiana, Tagus and Douro rivers) studied by Trigo et al. (2004).

From these results we can conclude that 4 atmospheric modes (NAO, SCAND, EA and EA/WR) are necessary to explain the variability structure of winter rainfall and river-flow regimes in Galicia. The influence of these patterns depends on the period studied and on the correlation between rainfall and river flow. The relationships between atmospheric circulation and river flow developed here aid our understanding of interannual variations in river flows in the region and allow for the potential to reconstruct gaps in river-flow measurements. Moreover, the possibility to provide hydrologists mechanisms by which they can forecast river flow (a few months in advance) for planning purposes is most interesting. The main drawback is that the atmosphere is a dynamical system with low predictability. However atmospheric teleconnection patterns are driven by internal atmospheric processes, but with external influences, such as sea-surface-temperature anomalies, ocean circulation or stratospheric coupling. A better understanding of these external influences and their roles in driving regional circulation would provide a measure of predictability in circulation regimes and therefore rainfall and river flow.
Acknowledgements. This work is a contribution to the Spanish LOICZ coordinate project METRIA supported by the CICYT under projects MAR96-1782 and REN2003-04106-C03 and by 'Xunta de Galicia' under project PGIDIT02PXJA38301PR. We acknowledge 'Confederacion Hidrografica del Norte' for providing Miño River data and L. Gimeno for his helpful discussions. Nieves Lorenzo acknowledges the support by the Ramon y Cajal Program.

\section{LITERATURE CITED}

Alexander LV, Jones PS (2001) Updated precipitation series for the UK and discussion of recent extremes. Atmos Sci Lett 1:142-150 (doi:10.1006/asle. 2000.0016)

Ambaum MHP, Hoskins BJ, Stephenson DB (2001) Arctic Oscillation or North Atlantic Oscillation? J Clim 14:3495-3507

Barnston AG, Livezey RE (1987) Classification, seasonality and persistence of low frequency atmospheric circulation patterns. Mon Weather Rev 115:1083-1126

Bojariu R, Gimeno L (2003a) The role of snow cover fluctuations in multiannual NAO persistence. Geophys Res Lett 30:1156

Bojariu R, Gimeno L (2003b) Predictability and numerical modelling of the North Atlantic Oscillation. Earth Sci Rev (doi:10.1016/S0012-8252(03)00036-9)

Cohen J, Entekhabi D (1999) Eurasian snow cover variability and Northern Hemisphere climate predictability. Geophys Res Lett 26:345-348

Cullen HM, Kaplan A, Arkin PA, DeMenocal PB (2002) Impact of the North Atlantic oscillation on the Middle Eastern climate and Streamflow. Clim Change 55:315-338

Czaja A, Marshall J (2002) Observed impact of Atlantic SST anomalies on the North Atlantic Oscillation. J Clim 15: 606-623

Esteban-Parra MJ, Rodrigo FS, Castro-Diez Y (1998) Spatial and temporal patterns of precipitation in Spain for the period 1880-1992. Int J Climatol 18:1557-1574

González-Rouco F, Zorita E, Heyen H, Valero F (2000) Agreement between observed rainfall trends and climate change simulations in the southwest of Europe. J Clim 13: 3057-3065

Goodess CM, Jones PD (2002) Links between circulation and changes in the characteristics of Iberian rainfall. Int $\mathrm{J}$ Climatol 22:1593-1615

Hurrell JW (1995) Transient eddy forcing of the rotational flow during northern winter. Atmos Sci 52:2286-2301

Jones PD, Lister DH (1998) Riverflow reconstructions for 15 catchments over England and Wales and an assessment of hydrologic drought since 1865. Int J Climatol 18: 999-1013

REE (1999-2004) Red Electríca Española. Available at www.ree.es

Rodó X, Baert E, Comin FA (1997) Variations in seasonal rainfall in southern Europe during the present century: relationships with the North Atlantic oscillation and the El Niño-southern oscillation. Clim Dyn 13:275-284

Rodríguez-Puebla C, Encinas AH, Nieto S, Garmendia J (1998) Spatial and temporal patterns of annual precipitation variability over the Iberian Peninsula. Int J Climatol 18:299-316

Rodríguez-Puebla C, Encinas AH, Sáenz J (2001) Winter precipitation over the Iberian Peninsula and its relationship to circulation indices. Hydrol Earth Syst Sci 5:233-244

Sáenz J, Zubillaga J, Rodríguez-Puebla C (2001) Interannual winter temperature variability in the north of the Iberian Peninsula. Clim Res 16:169-179 
Saunders MA, Budong Q (2002) Seasonal predictability of the winter NAO from north Atlantic sea surface temperatures. Geophys Res Lett 29:2049

Serrano A, García AJ, Mateos VL, Cancillo ML, Garrido J (1999) Monthly modes of variation of precipitation over the Iberian Peninsula. J Clim 12:2894-2919

Thompson DWJ, Wallace JM (1998) The Arctic Oscillation signature in the wintertime geopotential height and temperature fields. Geophys Res Lett 25:1297-1300

Thompson DWJ, Wallace JM (2000) Annular modes in the extratropical circulation. Part I: month-to-month variability. J Clim 13:1000-1016

Trigo RM, DaCamara CC (2000) Circulation weather types and their impact on the precipitation regime in Portugal. Int J Climatol 20:1559-1581

Editorial responsibility: Gregory V. Jones,

Ashland, Oregon, USA
Trigo RM, Pozo-Vázquez D, Osborn TJ, Castro-Díez Y, Gámiz-Fortis S, Esteban-Parra MJ (2004) North Atlantic Oscillation influence on precipitation, river flow and water resources in the Iberian Peninsula. Int J Climatol 24: 925-944

Wallace JM (2000) North Atlantic Oscillation/annular mode: two paradigms - one phenomenon. Q J R Meteorol Soc 126:791-805

Wallace JM, Gutzler DS (1981) Teleconnection in the geopotential height field during the Northern Hemisphere winter. Mon Weather Rev 109:784-812

Zorita E, Kharin V, von Storch H (1992) The atmospheric circulation and sea surface temperature in the North Atlantic area in winter: their interaction and relevance for Iberian precipitation. J Clim 5:1097-1108

Submitted: December 12, 2005; Accepted: May 18, 2006

Proofs received from author(s): July 11, 2006 